\title{
Factors that Hinder Students Benefitting from Videoconference Broadcast Services
}

\author{
Lesiba Molepo \\ Tuition and Facilitation of Learning, \\ Email: Lmolepo@unisa.ac.za \\ Hector Mothudi \\ Tuition and Facilitation of Learning, \\ Email: Hmothudi@unisa.ac.za
}

\section{Doi:10.5901/mjss.2014.v5n1p497}

\begin{abstract}
Unisa has over the years introduced videoconference discussion classes to support its students. However, there has been poor attendance of such classes by the students in Ekurhuleni regional service centre. A quantitative research through surveys was conducted with 88 students whose modules were offered via videoconference broadcast at Unisa's Ekurhuleni regional service centre during the first semester of 2012. The purpose of the study was to investigate the factors that hinder students from benefitting from videoconference broadcast services. It was found that many students did not attend these classes mainly because they did not receive notification about such classes. Some of those who received notification could not attend due to various reasons. The researchers found that there are three main factors that hinder students from benefitting from videoconference services - environmental, institutional and personal factors. It was found that there is not much Unisa can do about environmental factors. However, in order to mitigate institutional factors, the researchers recommend that Unisa introduce integrated and automated communication systems to inform students about videoconference discussion classes. Various technological software and adequate videoconference infrastructures are also recommended. With regard to personal factors, Unisa needs to increase students' awareness and utilization of other services such as counselling which are aimed at helping them cope with their studies.
\end{abstract}

Keywords: open and distance learning, videoconference, technology, facilitation of learning, discussion classes

\section{Introduction and Background}

The University of South Africa (Unisa) as an open and distance learning (ODL) institution is making increasing use of technology in order to reach and support the majority of its students as it was found that it would be impossible to reach and effectively support all Unisa students through face-to-face means. According to Prof. Mandla Makhanya, principal and vice-chancellor of Unisa, "Unisa is a large and complex organization that has been undergoing rapid transformation in terms of its policies, structures, systems, and use of technology, its capabilities and core pedagogies" (Makhanya, 2013). He presented a strong argument to the university council by stating that "a clear imperative is for the institutionwide introduction of an open distance and eLearning (ODeL) model" (Makhanya, 2013). The ODeL model incorporates a number of different types of technology communications (ICTs) in a university's learning environment (Gerbic, 2010:125).

In order to incorporate and advance the technological agenda, Unisa developed policies and adopted a business model that emphasised the integration of technology. It would seem that Taylor's (1995) assertion that there is an opportunity for institutional leaders to adopt a proactive stance and to generate an organisational development strategy which will lead to the new technologies becoming a structurally integrated element of the teaching/learning environment was correct. Unisa has since produced a strategic document in this regard, for instance the Regional Model document (Mashile, 2012). According to this document, "quality must be taken into consideration before any service is introduced in regional service centres". However, the researchers believe that it would be difficult to ensure quality services if basic issues such as the functionality of the existing technological devices are not addressed.

Unisa has various facilities and resources in its different centres to accommodate students who cannot access face-to-face services such as discussion classes. In fact, many face-to-face services are being phased out or scaled down within Unisa. Instead, other technological resources such as videoconferences are being used in an attempt to reach more students. Videoconferencing may be described as the transmission of image (video) and speech (audio) 
back and forth between physically separate locations (Dallas, 1999). Various discussions do take place between Unisa stakeholders through the use of videoconferencing. Lecturers too use videoconferencing specifically for academic discussion classes with students. This is in line with recommendation 11 of the Regional Model that states:

Videoconferencing should be maintained and developed further with opportunities for tutors in one region to support groups in another as well as central staff providing some teaching through this medium.

This recommendation is clearly embraced by the university's new vision to move towards the maximum use of technology. According to Gerbic (2010:125), in class discussions, students identify the value of the visual and aural cues which increases the depth and breadth of the information they receive. A benefit of videoconference discussions is that the interaction is synchronous.

The regional model further recommends that certain factors should be taken into consideration prior to the introduction of student support services in regional service centres. Such factors include (a) long-term availability of physical infrastructure, and (b) market research on the possible subscription to the service by students. These two factors are of relevance to Unisa's Ekurhuleni regional service centre where this study was conducted. This centre is situated outside Daveyton township, east of Johannesburg, and has been operational in this specific location since 2010.

This study looks at factors that hinder students from making use of videoconference discussion classes at Unisa's Ekurhuleni regional service centre. It specifically explores whether or not students receive notification about videoconference discussion classes. It then continues with the specific reasons why some students do not attend such classes despite received notifications. Finally, the article outlines the factors that hinder students from benefitting from videoconferencing and then recommends actions that can be taken for each category of factors.

\section{Problem Statement}

The researchers were concerned that despite the fact that videoconference discussion classes are offered free of charge by Unisa lecturers, very few students attend these classes even though the information to be shared at these classes is likely to be of relevance for the examination. Students usually sign an attendance register every time they attend a videoconference class, and this standard practice was used by the researchers to inspect the videoconference attendance registers of the first semester in 2013. It was found that students were not attending these classes as regularly as they should. There was also evidence of attrition. As a result of these findings, the researchers decided to undertake this study in order to probe the matter in more depth.

\section{The Aim of the Study}

This study aimed to investigate and ultimately recommend systems of proactive contact or interventions both from the advisory and teaching services (Simpson, 2004). It is the researchers' belief that once basic logistical issues are addressed and hopefully resolved, the focus can then shifts towards the monitoring of academic quality. The aim of the study was therefore to explore factors that hinder students from benefitting from the videoconference broadcast service at Unisa Ekurhuleni regional service centre.

\section{Objectives}

The objectives emanating from the aim of the study were:

- To investigate if students received notification about videoconference classes, and how they receive such notification?

- To investigate if students attended these classes

- To investigate if they are notified of the classes, why do they not attend?

- To identify the challenges experienced by those who attend the classes and suggestions to these challenges.

\section{Rationale}

This study hoped to contribute towards the development of relevant systems, procedures and infrastructure relating to videoconference offerings within Unisa in particular and to ODL contexts in general. It also wished to highlight the challenges experienced by students so that the students' voice could be heard and taken into consideration in future plans regarding videoconference endeavors. This is in line with the principle of student-centeredness which is one of the 
pillars of an ODL institution. It is believed that regional service centres have an obligation to share their experiences with the wider Unisa community and also to contribute to the broader ODL practices. Successes and challenges need to be shared so that coordinated and focused strategies can be put in place. The study can thus be viewed as a proactive intervention in that researchers took the initiative to contact students (Simpson, 2004) before the students dropped out of their studies.

\section{Research Hypothesis}

The research hypothesises that: There are several factors that hinder students from benefitting from videoconference broadcast service at Unisa Ekurhuleni regional service centre.

\section{Theoretical Framework}

The purpose of any theoretical framework is to provide guidance in determining what to look at and what needs to be examined (Mattaini, 2008:357). The researchers identified the ASSURE model in order to situate their study. This model is depicted in figure 1 below.

Figure 1. Assure Model (Heinich, Molenda, Russell and Smaldino, 1999)

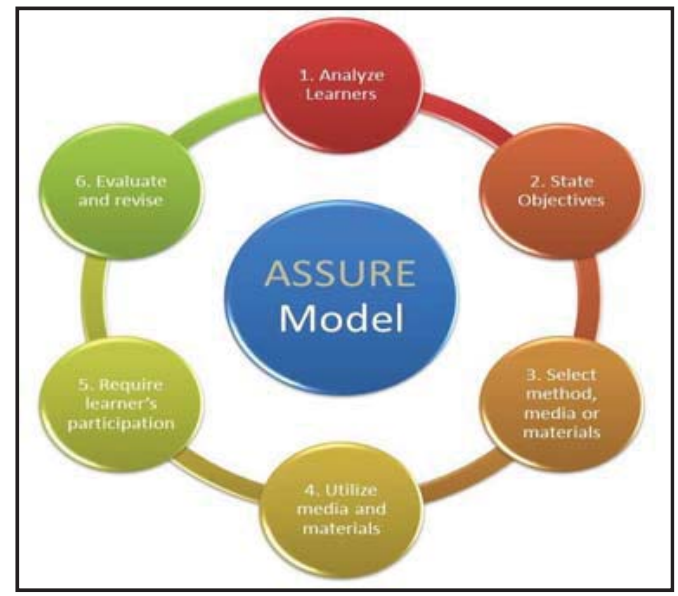

The ASSURE model was developed by Heinich, Molenda, Russell and Smaldino (1999) and is an instructional model for planning a lesson and the technology that will enhance it, and contains six steps.

The letters in ASSURE form an acronym. The "A" stands for "analyse" the learner, and answers the question: Who are our students?

The first "S" stands for "state objectives". Heinich et al. (1999) argue that the teacher will have a curriculum to teach in the classroom, with specific objectives that will become the focus of individual lessons. The researchers assumed that lecturers take care of this aspect when planning for a class.

The second "S" stands for "select media and materials". According to Heinich et al. (1999), when choosing the media and materials to help teach a lesson, one will first have to choose a method for delivering the instruction. One then selects the media that best supplements or enhances the chosen method of teaching. The media could include technology solutions (e. g. CD-ROMs, DVDs, calculators, software, Internet resources,videos), print resources such as a textbook, or any combination of the various media types. Within the ODL context, in addition to the selected media, lecturers have to add videoconferencing in order to accommodate the distant students.

The "U" stands for "utilize media and materials". In the last step one has to identify specific media and materials to help meet the learning objectives. In this step, the lesson is actually taught and the media and materials get implemented. Evaluating the media will also raise any red flags (e. g. Is any training going to be required? Is an extension cord going to be necessary?). In the ODL context whereby the broadcast is being transmitted via 
videoconference, lecturers are likely to take care of this point from a transmitting venue. They do, however, have no control of what is going on on the other side of the transmission venue.

The "R" stands for "require learner participation". Students find learning more meaningful when they are actively involved in the learning process and not sitting there passively. Are there strategies one can use to get students practically involved? Thinking? Solving? Creating? Developing? Analysing? More often than not, a lecturer is not going to allow students to move beyond passive learning. On the other hand, integrating technology into a lesson almost necessitates that lecturers use a teaching method beyond lecturing. The key question at this point is, how can the lecturer best facilitate the learning process? Once again, learner participation is regarded as one of the lecturer's priorities.

Finally, the "E" stands for "evaluate and revise". In order to be a good teacher, teachers must constantly evaluate their own teaching, evaluate the student learning, and make any revisions required for the next lesson and for the next time they are going to teach the lesson. Some of the key questions to be asked during evaluation are, "Was the media appropriate?", "Are there other technology solutions that might have worked better?"

All the components of the ASSURE model are relevant for the ODL context in general. Hence the researchers regarded this model a suitable framework to guide their study. It is the last $E$ that caught the researchers' attention in that it seems relevant to this study. In terms of the researchers' experiences, lecturers are not in a position to evaluate all aspects of the technological challenges experienced by the students. Regional staff members can therefore make a contribution by identifying and sharing their experiences. It is this kind of sharing that can result in improvements.

\section{Literature Review}

In Figure 2 below, Collis and Moonen (2004:8) outline four key components of flexible learning. These components are technology, pedagogy, implementation and the institution. In this study, the following three components are relevant: Technology, implementation and the institution. Because regional staff within Unisa is not involved in the development of course material, the component of pedagogy is excluded. However, this is not to say that this component is not important and relevant within the broader context of Unisa.

Figure 2. Four key components of flexible learning

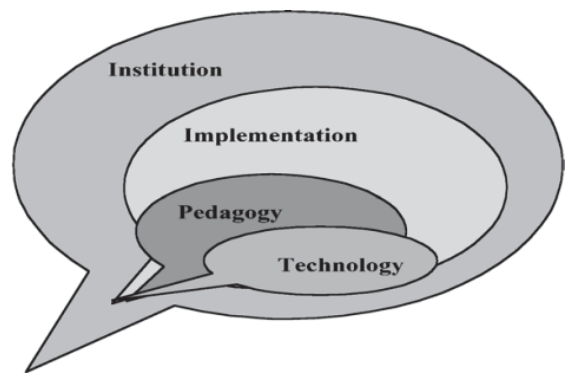

Although the components above speak specifically about flexible learning, they are very important in terms of the ODL context in which Unisa operates.

- Flexible learning is the key word in this model and is also a word that is used at Unisa. For flexibility to be adequately implemented at any institution, it has to be understood by all stakeholders of that institution. This flexibility has an impact on all staff, the systems as well as the resources of an institution.

- Flexible learning is often taken to mean the same as distance education. This is not necessarily the case. Flexibility can involve options in course resources, in types of learning activities, in media to support learning, and so forth. It is important that students are given a range of practical options to choose from in terms of their method of learning and learning resources. Students with various preferences need to be catered for as much as possible, taking into consideration all other factors.

In this study, the term "flexible learning" will be used in a broad way, with the key idea being learner choice in different aspects of the learning experience. 


\section{Research Methodology}

The aim of the study was such that the researchers had to adopt a quantitative research design. The researchers used a survey as this can also be used for both exploratory and descriptive studies (Fouche, Delport \& De Vos, 2011:156). Surveys are done mainly to describe some sample in terms of simple proportions and percentages of people with the view of tapping into matters of fact, preferences and opinions (Fouche et al., 2011:156).

\subsection{Population and Sampling}

The researchers were interested in all modules whose discussion classes had been offered via video conferencing (VC) within Unisa's regional service centres. These modules therefore served as the population that set boundaries to the study unit (Strydom, 2011: 223). However, given that not all modules could be selected, random sampling was done to identify the modules that could be included in this study. There were 13 modules that were offered via videoconference within Unisa during this particular semester. Thus all those modules that were offered at Unisa's Ekurhuleni regional service centre via videoconferencing were purposely selected.

With regard to the respondents, there were about 300 students who were registered at Unisa's Ekurhuleni regional service centre and whose modules were offered via videoconference. Researchers used structured English query language (SQL), a data management system, to generate a list of registered students in the identified modules. From this SQL generated list, 88 students were randomly selected as a sample, a trend that is in line with Stoker's guidelines for sampling (Stoker, 1985).

\subsection{Data Collection Method}

A structured questionnaire was developed by the researchers for data collection purposes. Students registered at Unisa are given a standardised Unisa myLife e-mail address, regardless of whether they have their own e-mail or not (Roberts, 2011:37). The logical expectation would be for the researchers to use technology-related tools such as email to distribute the questionnaires. However, according to Roberts (2011:38), the majority of students do not have access to or are unable to open and access their Unisa myLife e-mail addresses. The researchers therefore opted for telephone interviews which guaranteed quick responses. Telephone interviews where thus conducted with all the relevant students to administer this questionnaire. According to Delport and Roestenburg (2011:186), "the basic objective of a questionnaire is to obtain facts and opinions about a phenomenon from people who are informed about a particular issue". As the answers were made, they were recorded on the questionnaire (Maree \& Pietersen, 2007:157).

The telephonic survey was relevant given the ODL nature of Unisa. The response rate was high and researchers were able to collect data quickly (Delport \& Roestenburg, 2011:187).

Table 1 below will be used as the basis for interpreting the findings.

Table 1. Respondents' answers to the questionnaire

\begin{tabular}{|c|c|c|c|}
\hline Total no. of modules & \multicolumn{3}{|c|}{13} \\
\hline No. of students contacted & \multicolumn{3}{|c|}{88} \\
\hline Notified about VC class & 24 & Via SMS (21) & Via email (3) \\
\hline Not notified about VC class & \multicolumn{3}{|c|}{64} \\
\hline Attended VC class & \multicolumn{3}{|c|}{09} \\
\hline Did not attend VC class & \multicolumn{3}{|c|}{79} \\
\hline
\end{tabular}

\section{Analysis and Discussion of Results}

\subsection{Research results in relation to the analysing of students}

The researchers used descriptive methods to produce the scope of the characteristics of distribution through frequencies (Fouche \& Bartley, 2011:251). Within Unisa, students are notified about VC discussion classes via short message services (SMS) and email. It was found that the majority of students (21) obtained the notification about the videoconference discussion classes via SMS as compared to three who received notification via email. It would seem 
that the cellphone is the most accessible and/or preferred tool for communication for most students. Despite the fact that Unisa offers all students an email address, it seems students have their own preference in terms of how to access communication from the institution. This is an area that needs to be researched further. It would certainly be beneficial for Unisa to take cognisance of the most preferred mode of communication if it wishes to maximise its communication with its students.

\subsubsection{Notification of VC classes}

The researchers focused on 13 modules to conduct this study. From Table 1 above, only 24 out of 88 students received notifications about VC classes. This is clearly of concern as it seems the communication strategies used to notify students are ineffective. Unisa cannot convincingly claim to offer support systems if the majority of students do not receive the notifications about such services.

\subsubsection{Reasons for non-attendance}

Table 1 also shows that only nine students out of 88 attended the video conference classes. This too is cause for concern. Students cited a variety of reasons for not attending the VC classes. Reasons included that the Unisa regional service centre was located too far from where they stay, not knowing about the VC classes, work commitments, lack of public transport after hours, late notification about classes as well as being hospitalised. In terms of the ASSURE model, lecturers should know who their students are (e. g. demographics, prior knowledge, learning styles, academic abilities) on a number of levels, and use this knowledge in every lesson plan. Within the Unisa context it is not always possible for lecturers to know the precise demographics of their students although Unisa does have a system that generates an overview of biographic information of students.

One student mentioned that he did not read the message as he received too many messages from Unisa - an issue that may need further investigation. It might be that Unisa staffs are overusing this mode of communication, and thus overwhelming students to such an extent with messages that the students are ignoring and overlooking some of the important academic communications. On the other hand, students might not be blameless either, as lecturers might believe that more communication is better than no or less communication. According to Simpson (2004), any contact must clearly catch the students' attention and be seen to be relevant to their needs. It is important for Unisa staff to communicate about administrative as well as academic matters. Case and Elliot (1997) in Simpson (2004) found that between two and five telephone contacts seemed to be more effective. At the moment, there is no doubt that Unisa sends far more messages than recommended by the authors mentioned above.

\subsection{Research results in relation to the selected media}

\subsubsection{Challenges experienced by those who attended}

Students who have managed to attend VC classes also cited some problems experienced during the actual broadcasts. One of the problems is that at times there is no clear signal coverage resulting in some break-ups. As a result the students cannot always follow the discussions. Students also mentioned that the television screens used in the VC room are too small and they thus cannot see drawings and measurements clearly on the screens. These students felt that coming for further classes would be "a waste of time and petrol". This sentiment clearly translates to a bad student experience.

\subsection{Conclusion and discussion of overall results}

There are several factors that hinder students from benefitting from videoconference broadcasts. These factors can be classified into the following three categories: Environmental factors, institutional factors and personal factors. Below is a brief discussion of each of these factors.

\subsubsection{Environmental factors}

Environmental factors are those that have to do with the location of the centre. Ekurhuleni regional service centre is 
situated outside Daveyton. Although the centre is accessible to those who have their own transport, it is not easily accessible to those using public transport, especially after hours. The signal bandwidth in this particular area is also very poor. It would require a lot of investment not only from Unisa but also from the government to improve this situation. These are factors that are therefore out of Unisa's control. It is important to note that the grounds and buildings where the centre is located are not owned by Unisa, which will make it difficult to justify major infrastructural investments in this area.

\subsubsection{Institutional factors}

Although Simpson (2004) observed that some drop-out is beyond the power of any institution to influence, the same can be said about non-attendance of some services. The findings of this study clearly show that the method that Unisa uses to communicate with students about the availability of videoconference classes is not effective. It is suspected that the SMSs that are sent to students do not reach most of them. This, of course, may be due to a number of different reasons such as students having changed their telephone numbers or not checking their messages. By the very nature of ODL, it is not always possible to accommodate all the students' needs. Scheduling of broadcasts is a classical example. Unisa schedules most of its videoconference classes during weekdays, an arrangement which obviously does not accommodate the majority of working students. In order to accommodate employed students, there are times when Unisa schedules classes either in the evenings or during weekends. However, the evening sessions will also not be suitable for those students who do not have their own transport.

\subsubsection{Inadequate infrastructure}

Dallas (1999) mentions that when using videoconferencing technology, two or more persons at different locations must be able to see and hear each other at the same time. Some of the problems cited by the students relate to infrastructure. Synchronous communication is supposed to allow feedback and interaction as well as flowing discussions which are more productive and better for the development of ideas (Gerbic, 2010:131). The bandwidth at Unisa's Ekurhuleni regional service centre is generally not adequate. The videoconference equipment is connected to the internet protocol (IP) which becomes overloaded when other services are up and running. During the videoconference broadcasts, the quality of pictures and sound is generally poor. The ASSURE model acknowledges the imminent breakdown of technologies. Hence the suggestion that alternative solution should be available (Heinich et al., 1999).

Although in terms of the ASSURE model, a teacher should always test the media first to prevent disappointment, within Unisa and probably other ODL contexts, this is not always the sole responsibility of the lecturer. In some instances, there are dedicated staff members whose responsibility is to test the main videoconference equipment. From the perspective of the regional service centre, staff members are mainly tasked with the responsibility of switching on the main devices. These staff members do not necessarily have the technical knowhow of trouble shooting in the event of an unexpected technical glitch. They in turn rely solely on the staff members on the main campus. These breaks make it difficult for students to follow discussions and to attract those who make an effort to attend in order to experience these technological innovations. Although Taylor (1995) contends that what really matters is the quality of the instructional message, rather than any inherent characteristics of the instructional medium used, the researchers believe that the medium is equally important. What is the point of using a medium that cannot transmit the instructional message one wants to transmit? There is thus a gap in the ASSURE model in that it does not emphasise the evaluation of technical equipment by technically trained people.

\subsubsection{Personal factors}

Personal circumstances also hinder some students' from benefitting from videoconference broadcasts. Simpson (2004) cited examples of illness, domestic and employment circumstances and other factors interacting in various ways. These are indeed some of the reasons mentioned by the respondents. According to Artino (2008:39), self-regulated learners are generally characterized as active participants who efficiently control their own learning experiences in many different ways, including establishing a productive work environment and using resources effectively. From the responses received, it seems the majority of Unisa students struggle with self-regulation as described by Artino (2008). Some students mentioned that they could not attend the videoconference classes due to work commitments. This is an area that Unisa has no control over. ODL students are expected to balance their studies with other commitments. They are 
also at liberty to priorities activities. It is thus easy to rush to the conclusion that it is unfortunate that some activities in the students' lives take precedence over their studies, and there is thus not much Unisa can do. However, according to Gerbic (2010:125), courses and teachers should be sufficiently flexible to enable everyone to learn. Unisa, just like many other ODL university, can therefore not be exonerated from its responsibility of ensuring that every student is given an opportunity to learn.

Some students in the study said that they felt that they were coping with their studies and therefore saw no need to attend additional classes. Unisa has students from different backgrounds. Some of these students have attended good secondary schools which have given them the confidence and ability to cope with their tertiary studies. Such students may see no necessity to make use of whatever student support services Unisa puts in place. After all, student support services generally target those students who are not coping with their studies. It is, however, of concern that some students may think that they are coping, only to be surprised at the end of the academic year when they are faced with difficult examination papers, resulting in them failing their modules. Learning at a tertiary institution can be far more demanding and also different from high school learning. Hence overconfidence by any student should be treated with concern. Taylor (1995) cites "proactive motivational support" as the factor that can improve students' retention. By the time such students realize that they actually do need the student support services, it will most likely be too late. All students should therefore be encouraged to participate in student support initiatives that suit their preferences and situation.

\section{Recommendations}

In its business model document, Unisa (2013) identified "heavy investment by Unisa in its ICT infrastructure" as an opportunity, i.e. Unisa should consider all the current and future technologies when making such investments. During this investment period various information communication technologies (ICTs) have to be included to support the new and envisaged learning environments (Gerbic, 2010:125). The researchers of this study thus make the following recommendations:

\subsection{Integrated and automated communication system}

As Unisa is moving towards investing in technologies, some of these technologies should be geared towards improving communication. It is recommended that an integrated and automated communication system should be put in place that will replace the current manual SMS system. Such a system should, for instance, be able to notify students as soon as a booking is made, and should also be able to notify them of any changes, updates and cancellations.

Although videoconferences are synchronous in nature, there might be a need for Unisa to consider other asynchronous options for those who cannot attend live broadcasts. An asynchronous online discussion environment offers participants (e.g. students and instructors) the flexibility and convenience of communicating with one another without the constraint of time and geographical location (Hewet al., 2010). The ASSURE model requires educators to constantly keep up to date with new and emerging technologies that might be more effective (Heinchil et al., 1999). Podcasts are examples of such asynchronous technologies.

\subsection{Skype link, podcast and/or live stream}

According to Borup, West and Graham (2013:52), new technological advances have allowed online courses to use asynchronous video communication. If Unisa wishes to continue using the current video conference equipment, it should at least implement bigger screens such as projected screens as are already fitted in some centres. The systematic introduction of podcasts alongside videoconference broadcasts might also be a valuable addition to accommodate the diverse needs of Unisa students. The use of a Skype link is also worth considering. Although this service might be costly, it is worth exploring as it is likely to get cheaper in the near future with the introduction of more technologies. According to Dallas (1999:292), technology for conducting videoconferencing has become less expensive, more flexible, and now includes options for desktop video conferencing as well as group videoconferencing. Unisa can also explore the live stream avenue whereby students would be able to view these classes from the comfort of their own homes or offices. This method will come in handy for those students who are always under time pressure due to various commitments. 


\section{Conclusion}

There are many technological devices that can be recommended for the ODL environment. However, those suggested here are merely as a result of their closeness to the videoconference offerings. There are several other factors that Unisa needs to pay attention to in order to effectively support its students.

\section{Limitations of the Study}

The sample was not big enough for the researchers to be able to generalize the results across the entire Unisa student population. However, the results do provide a clear picture of what is happening at Unisa's Ekurhuleni regional service centre.

The study was carried out before the policy on obtaining ethical clearance when conducting research on Unisa students and staff was passed in 2012. Thus, before the findings could be presented at a seminar, this policy was passed. According to the Unisa research guidelines, Permission to conduct research involving Unisa staff, students and/or data must be sought from the Senate Research and Innovation Committee (SENRIC) before any research can be carried out (Unisa, 2012). Since these guidelines were introduced after the research was carried out, the researchers subsequently informed the Professional and Administrative Staff Research Committee (PARC) Ethics Committee about how it carried out this research. The committee expressed its satisfaction about the manner in which the research was carried out.

\section{References}

Artino, A.R. (2008). Promoting academic motivation and self-regulation: Practical guidelines for online instructors. TechTrends, 52(3), 37 -45 .

Borup, J., West, R.E. \& Graham, C.R. (2013). The influence of asynchronous video communication on learner social presence: a narrative analysis of four cases. Distance Education, 34:1, 48-63.

Cresswell, J.W. (2007). Qualitative enquiry and research design: Choosing among five approaches. London: SAGE.

Dallas, P.S. (1999). Video conferencing application to distance education with particular

Reference to small states. Available http://www.col.org/SiteCollectionDocuments/2_conf_proc_Dallas.pdf (Accessed 2013/04/22).

Collis, B. \& Moonen, J. (2004). Flexible learning in a digital world: experiences and expectations. Oxon: RoutledgeFalmer.

Delport, C.S.L. \& Roestenburg, W.J.H. (2011). Quantitative data-collection methods: questionnaires, checklists, structured observation and structured interview schedules. In De Vos, A.S., Strydom, H., Fouché, C.B. \& Delport, C.S.L. (Eds). Research at grassroots for the social sciences and human service professions. 4th ed. Pretoria: Van Schaik.

Gerbic, P. (2010). Getting the blend right in new learning environments: A complementary approach to online discussions. Educ Inf Technol, 15:125-137.

Heinich, R., Molenda, M., Russell, J.D \& Smaldino, S.E. (1999). ASSURE MODEL Available at: http://ed205.net/assure_model.html (Accessed 2013/07/22).

Maree, K. \& Pietersen, J. (2007). The quantitative research process. In Maree, K., First steps in research. Pretoria: Van Schaik.

Mashile, E. O. (2012). Regional Model. Pretoria: Unisa

Roberts, J.J. (2011). Biblical archaeo-tourism: A new vocational opportunity for biblical archaeology students. (Unpublished doctoral thesis), South Africa: University of South Africa.

Simpson, O. (2004). The impact on retention of interventions to support distance learning students. Open Learning, 19(1), 79 - 95.

Stoker, D.J. (1985). Sampling. Personal communication to the author. Pretoria: Human Sciences Research Council.

Strydom, H. (2011). Sampling in the quantitative paradigm. In De Vos, A.S., Strydom, H., Fouché, C.B. \& Delport, C.S.L.(Eds). Research at grassroots for the social sciences and human service professions. 4th ed. Pretoria: Van Schaik.

Taylor, J.C. (1995). Distance education technologies: The fourth generation. Australian Journal of Educational Technology, 11(2), 1-7. 
